\title{
Nucleon generalized form factors from lattice QCD with nearly physical quark masses
}

\author{
Gunnar Bali, ${ }^{a}$ Sara Collins, ${ }^{a}$ Meinulf Göckeler, ${ }^{a}$ Rudolf RödI ${ }^{* a}$ Andreas Schäfer, ${ }^{a}$ \\ Andre Sternbeck ${ }^{b}$ \\ ${ }^{a}$ Institut für Theoretische Physik, Universität Regensburg, 93040 Regensburg, Germany \\ ${ }^{b}$ Theoretisch-Physikalisches Institut, Friedrich-Schiller-Universität Jena, 07743 Jena, Germany \\ E-mail: Rudolf.Roedlephysik.uni-regensburg.de
}

\begin{abstract}
We determine generalized form factors of the nucleon from lattice simulations with $N_{f}=2$ massdegenerate non-perturbatively improved Wilson-Sheikholeslami-Wohlert fermions down to a pion mass of $150 \mathrm{MeV}$. We also present the resulting isovector quark angular momentum. Possible excited-state contaminations are investigated with correlated simultaneous fits.
\end{abstract}

The 33rd International Symposium on Lattice Field Theory

14 -18 July 2015

Kobe International Conference Center, Kobe, Japan*

\footnotetext{
*Speaker.
} 


\section{Introduction}

Generalized Parton Distributions (GPDs) were introduced in the '90s [1, 2]. They describe the inner structure of the nucleon. The starting point is the parametrization of the off-diagonal matrix elements of the light cone operator $[3,4,5,6]$

$$
\begin{array}{r}
\left\langle N\left(P^{\prime}\right)\left|\mathscr{O}_{q}(x)\right| N(P)\right\rangle \stackrel{\text { twist } 2}{=} \frac{1}{2} \bar{u}\left(P^{\prime}\right)\left[H_{q}(x, \xi, t) \not h+E_{q}(x, \xi, t) \frac{\mathrm{i} \sigma^{\mu v} n_{\mu} \Delta_{v}}{2 m_{N}}\right] u(P), \\
\mathscr{O}_{q}(x)=\frac{1}{2} \int_{-\infty}^{+\infty} \frac{\mathrm{d} \lambda}{2 \pi} \mathrm{e}^{\mathrm{i} \lambda x} \overline{\psi_{q}}\left(-\frac{\lambda}{2} n\right) \not h \mathscr{P} \exp \left[-\mathrm{i} g \int_{-\frac{\lambda}{2}}^{+\frac{\lambda}{2}} \mathrm{~d} \alpha A^{\beta}(\alpha n) n_{\beta}\right] \psi_{q}\left(+\frac{\lambda}{2} n\right) .
\end{array}
$$

The path ordering operator $\mathscr{P}$ in eq. (1.2) ensures gauge invariance. Further, it depends on the momentum fraction $x$ and the quark flavor $q$. The spinors $\bar{\psi}(\cdot)$ and $\psi(\cdot)$ are parametrized along the light cone $\left(n^{2}=0\right)$ by the parameter $\lambda$.

Key ingredients of the parameterization of the matrix element in eq. (1.1) are the constraint given by the Lorentz structure and the definitions of $H_{q}(\cdot)$ and $E_{q}(\cdot)$, which depend on the momentum fraction $x$ and the kinematic variables $\xi=-n^{\mu} \Delta_{\mu} / 2$ and $t=\Delta^{2}$ with $\Delta^{\mu}=P^{\prime \mu}-P^{\mu}$.

The direct calculation of GPDs by virtue of lattice QCD is not possible since $\mathscr{O}_{q}(x)$ is a light cone operator. However, one can expand eq. (1.2) in towers of local twist two operators

$$
\begin{aligned}
&\left\langle N\left(P^{\prime}\right)\left|\bar{\psi}_{q} \gamma^{\{\mu} \mathrm{i} \stackrel{\leftrightarrow}{D} \cdots \mathrm{\mu _{1 }} \cdots \stackrel{\leftrightarrow}{D}^{\left.\mu_{n}\right\}} \psi_{q}\right| N(P)\right\rangle= \\
&= \bar{u}\left(P^{\prime}\right)\left\{\sum_{i=0}^{n} \delta_{i, \text { even }} \gamma^{\{\mu} \Delta^{\mu_{1}} \cdots \Delta^{\mu_{i}} \bar{P}^{\mu_{i+1}} \cdots \bar{P}^{\left.\mu_{n}\right\}} A_{n+1, i}^{q}(t)\right. \\
&+\frac{-\mathrm{i}}{2 m} \sum_{i=0}^{n} \delta_{i, \text { even }} \Delta_{\alpha} \sigma^{\alpha\{\mu} \Delta^{\mu_{1}} \cdots \Delta^{\mu_{i}} \bar{P}^{\mu_{i+1}} \cdots \bar{P}^{\left.\mu_{n}\right\}} B_{n+1, i}^{q}(t) \\
&\left.+\frac{1}{m} \delta_{n, \text { odd }} \Delta^{\mu} \cdots \Delta^{\mu_{n}} C_{n+1}^{q}(t)\right\} u(P)
\end{aligned}
$$

The coefficient functions $A_{n+1, i}^{q}(t), B_{n+1, i}^{q}(t)$ and $C_{n+1}^{q}(t)$ are so called (vector) Generalized Form Factors (GFFs) which are related to (vector) GPDs by

$$
\begin{aligned}
\int_{-1}^{+1} \mathrm{~d} x x^{n} H^{q}(x, \xi, t) & =\sum_{i=0}^{n} \delta_{i, \text { even }}(-2 \xi)^{i} A_{n+1, i}^{q}(t)+\delta_{n, \text { odd }}(-2 \xi)^{n+1} C_{n+1}^{q}(t), \\
\int_{-1}^{+1} \mathrm{~d} x x^{n} E^{q}(x, \xi, t) & =\sum_{i=0}^{n} \delta_{i, \text { even }}(-2 \xi)^{i} B_{n+1, i}^{q}(t)-\delta_{n, \text { odd }}(-2 \xi)^{n+1} C_{n+1}^{q}(t) .
\end{aligned}
$$

The knowledge of $A_{2,0}^{u-d}(t), B_{2,0}^{u-d}(t)$ is of particular interest since they encode the total angular momentum

$$
J^{u-d}=\frac{1}{2}\left(A_{2,0}^{u-d}(t=0)+B_{2,0}^{u-d}(t=0)\right) .
$$

Moreover, we determine $\tilde{A}_{2,0}^{u-d}(t)$ and $\tilde{B}_{2,0}^{u-d}(t)$ which are the GFFs corresponding to the axial GPDs. 


\section{Lattice set-up}

We use $N_{f}=2$ mass-degenerate non-perturbatively improved Wilson-Sheikholeslami-Wohlert fermions with Wilson glue. The necessary gauge configurations were produced by the QCDSF collaboration and RQCD (Regensburg QCD). The lattice spacing was set as described in [7]. To ensure ground-state dominance we use optimized Wuppertal smearing as described in [8]. All results are computed at a renormalization scale $\mu=2 \mathrm{GeV}$ using non-perturbatively improved conversion factors. In table 1 we give an ensemble overview.

\begin{tabular}{|c|c|c|c|c|c|c|c|c|}
\hline Ensemble & $\beta$ & $a[\mathrm{fm}]$ & $\kappa$ & $V$ & $m_{\pi}[\mathrm{GeV}]$ & $L m_{\pi}$ & $N_{\text {conf }}$ & $t_{\mathrm{f}} / a$ \\
\hline I & 5.20 & 0.081 & 0.13596 & $32^{3} \times 64$ & $0.2795(18)$ & 3.69 & 1986(4) & 13 \\
\hline II & 5.29 & 0.071 & 0.13620 & $24^{3} \times 48$ & $0.4264(20)$ & 3.71 & $1999(2)$ & 15 \\
\hline III & & & 0.13620 & $32^{3} \times 64$ & $0.4222(13)$ & 4.90 & $1998(2)$ & 15,17 \\
\hline IV & & & 0.13632 & $32^{3} \times 64$ & $0.2946(14)$ & 3.42 & $2023(2)$ & $\begin{array}{c}7(1), 9(1), 11(1) \\
13,15,17\end{array}$ \\
\hline$\square \mathrm{V}$ & & & & $40^{3} \times 64$ & $0.2888(11)$ & 4.19 & $2025(2)$ & 15 \\
\hline VI & & & & $64^{3} \times 64$ & $0.2895(07)$ & 6.71 & $1232(2)$ & 15 \\
\hline VII & & & 0.13640 & $48^{3} \times 64$ & $0.1597(15)$ & 2.78 & $3442(2)$ & 15 \\
\hline VIII & & & & $64^{3} \times 64$ & $0.1497(13)$ & 3.47 & 1593(3) & $9(1), 12(2), 15$ \\
\hline IX & 5.40 & 0.060 & 0.13640 & $32^{3} \times 64$ & $0.4897(17)$ & 4.81 & $1123(2)$ & 17 \\
\hline $\mathbf{X}$ & & & 0.13647 & $32^{3} \times 64$ & $0.4262(20)$ & 4.18 & $1999(2)$ & 17 \\
\hline$\square \mathrm{XI}$ & & & 0.13660 & $48^{3} \times 64$ & $0.2595(09)$ & 3.82 & $2177(2)$ & 17 \\
\hline
\end{tabular}

Table 1: $N_{f}=2$ lattice set-up. Number of sources per configuration in brackets.

\section{Extracting Generalized Form Factors}

GFFs are obtained by solving an (in general) overdetermined system of equations. In the case of the vector GFFs the equation system reads

$$
\varepsilon\left(t, \tau_{\text {sink }}\right)=\left[\mathrm{M}\left[\begin{array}{c}
A_{2,0}(t) \\
B_{2,0}(t) \\
C_{2}(t)
\end{array}\right]-\vec{c}\left(t, \tau_{\text {sink }}\right)\right]^{T} \operatorname{cov}^{-1}\left(\vec{c}\left(t, \tau_{\text {sink }}\right)\right)\left[\mathrm{M}\left[\begin{array}{c}
A_{2,0}(t) \\
B_{2,0}(t) \\
C_{2}(t)
\end{array}\right]-\vec{c}\left(t, \tau_{\text {sink }}\right)\right] .
$$

We extract the GFFs of interest by minimizing eq. (3.1). The coefficient matrix $M$ in eq. (3.1) is fully determined by eq. (1.3). The matrix elements $\vec{c}\left(t, \tau_{\mathrm{sink}}\right)$ are extracted from lattice three-point functions. The dependency on the source-sink separation $\tau_{\text {sink }}{ }^{1}$ is examined in the next section, where in the limit $\tau_{\text {sink }} \rightarrow \infty$ the ground state GFFs are obtained.

A good signal is a prerequisite for excited-state fits since we have to fix many fit parameters. It turns out that excited-state fits are not possible for $t<0$ if we naively implement eq. (3.1). However, we can dramatically improve the signal if we average three-point functions which lead to the same rows in $M$.

\footnotetext{
${ }^{1}$ We set $\tau_{\text {source }}=0$.
} 


\section{Extraction of matrix elements from lattice QCD and excited states.}

As mentioned in section 2 we use optimized Wuppertal smearing on our quark field interpolators. Possible remaining excited states contributions are treated with simultaneous combined fits to two- and three-point functions. We therefore parametrize the two and three-point functions

$$
\begin{aligned}
C_{3}\left(\vec{p}_{i}, \tau, \tau_{\text {sink }}\right) & =c\left(\tau_{\text {sink }}\right) \sqrt{z_{N}^{0} z_{N}^{\vec{p}_{i}}} \mathrm{e}^{-m_{N}\left(\tau_{\text {sink }}-\tau\right)} \mathrm{e}^{-E\left(\vec{p}_{i}, m_{N}\right) \tau} \\
& +x_{1} \mathrm{e}^{-m_{N}\left(\tau_{\text {sink }}-\tau\right)} \mathrm{e}^{-E^{\prime}\left(\vec{p}_{i}\right) \tau}+x_{2} \mathrm{e}^{-m_{N}^{\prime}\left(\tau_{\text {sink }}-\tau\right)} \mathrm{e}^{-E\left(\vec{p}_{i}, m_{N}\right) \tau}+x_{3} \mathrm{e}^{-m_{N}^{\prime}\left(\tau_{\text {sink }}-\tau\right)} \mathrm{e}^{-E^{\prime}\left(\vec{p}_{i}\right) \tau}, \\
C_{2}^{1 e}(p, \tau) & =z_{N}^{\vec{p}} \frac{E\left(\vec{p}, m_{N}\right)+m_{N}}{E\left(\vec{p}, m_{N}\right)} \mathrm{e}^{-E\left(\vec{p}, m_{N}\right) \tau}, \\
C_{2}^{2 e}(p, \tau) & =z_{N}^{\vec{p}} \frac{E\left(\vec{p}, m_{N}\right)+m_{N}}{E\left(\vec{p}, m_{N}\right)} \mathrm{e}^{-E\left(\vec{p}, m_{N}\right) \tau}+z_{N^{\prime}}^{\vec{p}} \frac{E^{\prime}(\vec{p})+m_{N^{\prime}}}{E^{\prime}(\vec{p})} \mathrm{e}^{-E^{\prime}(\vec{p}) \tau} .
\end{aligned}
$$

Above we denote the nucleon mass as $m_{N}$ and its energy as $E\left(\vec{p}, m_{N}\right)$. Further, we assume the continuum dispersion relation for the ground state $E\left(\vec{p}, m_{N}\right)=m_{N}^{2}+\vec{p}^{2}$. All expressions corresponding to the first excited-state are indicated by a prime. The energy of the first excited-state $E^{\prime}(\vec{p})$ is left as a fit parameter since this could be a mulit-hadronic state. Our kinematic set-up is chosen such that the final momentum is always zero $\vec{p}_{f}=\overrightarrow{0}$. To accomplish the ground state extraction (parameter $c\left(\tau_{\text {sink }}\right)$ ) we fix $z_{N}^{\overrightarrow{0}}$ and $z_{N}^{\vec{p}_{i}}$ by virtue of the two-point functions.

\section{Assessment of the excited-state fit}

We simultaneously fit all parameters according to the coefficient matrix $M$. This is not trivial but has the advantage that rows with a poor signal are stabilized by others. The fit parameter $x_{3}$ in eq. (4.1) is only resolvable if multiple $\tau_{\text {sink }}$ per ensemble are available. Therefore, we perform in general a 3-exponent fit where we set $x_{3}=0$. To check whether this is justified or not we utilize ensemble VIII which has three different source sink separations $\tau_{\text {sink }} / a=9,12,15$, see fig. 1 .

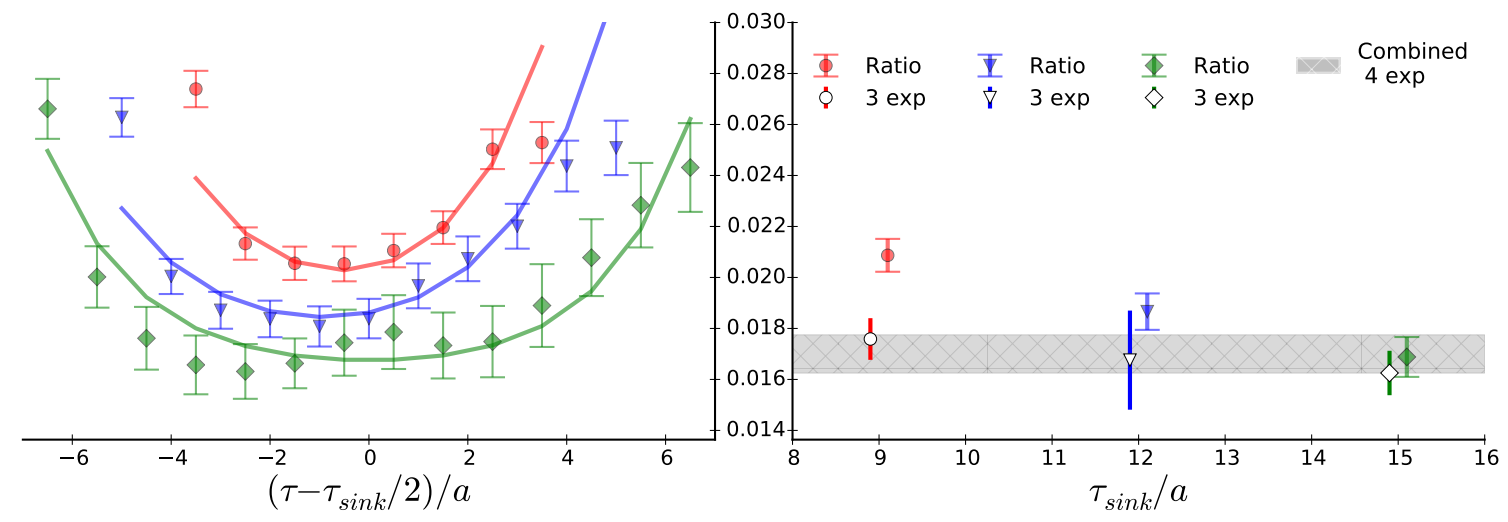

Figure 1: Extraction method analysis: Data points in the left panel are ratios of three-point and two-point functions for different source sink separations of ensemble VIII for a certain row $r_{1}$ in the coefficient matrix $M$ at $t=-0.211 \mathrm{GeV}^{2}$. The corresponding solid lines are ratios created with the fit functions using eq. (4.1) and setting $x_{3}=0$. Right panel shows matrix elements $c\left(\tau_{\text {sink }}\right)$ obtained with different extraction methods. 
In the right panel of fig. 1 we compare different values for the matrix element as a function of $\tau_{\text {sink }}$ and as a function of different extraction methods. More specifically, we compare the ratio method and the 3 exponent excited-state fit to the full excited-state fit with 4 exponents which is shown as a gray band.

We conclude from the right panel that at given statistics a distinction between the different fit methods is not possible if the source sink separation is $\tau_{\text {sink }} / a=15$ which is about $1 \mathrm{fm}$. This is consistent with what we have found in [8]. In this work we use the 3-exponent fit.

\section{Extraction of $A_{2,0}^{u-d}, B_{2,0}^{u-d}$ and $J^{u-d}$}

We utilize Baryon Chiral Perturbation Theory to extract $J^{u-d}$ at physical pion mass. Our aim is to quote an upper and lower bound of $A_{2,0}^{u-d}, B_{2,0}^{u-d}$ and $J^{u-d}$ including the statistical error and the systematical error from the fit range choice. To that end we perform global combined fits to our lattice ensembles with fit functions $A_{2,0}^{u-d}\left(t, m_{\pi}^{2}, \vec{\Theta}_{A}\right)$ and $B_{2,0}^{u-d}\left(t, m_{\pi}^{2}, \vec{\Theta}_{B}\right)$ known from (BChPT) [9]. More specifically,

$$
\begin{aligned}
& A_{2,0}^{u-d}\left(t, m_{\pi}\right)=\left[1-\frac{\left(1+3 g_{A}^{2}\right) m_{\pi}^{2} \log \left(\frac{m_{\pi}^{2}}{\mu^{2}}\right)}{16 f_{\pi}^{2} \pi^{2}}\right] L+m_{\pi}^{2} M_{2}^{A}+m_{\pi}^{3} M_{3}^{A}+t\left(T_{0}^{A}+m_{\pi}^{2} T_{1}^{A}\right) \\
& B_{2,0}^{u-d}\left(t, m_{\pi}\right)=\frac{g_{A}^{2} m_{\pi}^{2} \log \left(\frac{m_{\pi}^{2}}{\mu^{2}}\right)}{16 f_{\pi}^{2} \pi^{2}} L+\left[1-\frac{\left(1+2 g_{A}^{2}\right) m_{\pi}^{2} \log \left(\frac{m_{\pi}^{2}}{\mu^{2}}\right)}{16 f_{\pi}^{2} \pi^{2}}\right] L^{B}+m_{\pi}^{2} M_{2}^{B}+t\left(T_{0}^{B}+m_{\pi}^{2} T_{1}^{B}\right) .
\end{aligned}
$$

with fit parameters $\vec{\Theta}_{A}=\left(L, M_{2}^{A}, M_{3}^{A}, T_{0}^{A}, T_{1}^{A}\right)$ and $\vec{\Theta}_{B}=\left(L, L^{B}, M_{2}^{B}, T_{0}^{B}, T_{1}^{B}\right)$. The fit parameters $T_{1}^{A}$ and $T_{1}^{B}$ are introduced by hand but they naturally appear in next order of BChPT. We do this since we consider relatively large virtualities with $\left|t_{\max }\right|=0.6 \mathrm{GeV} \gg m_{\pi}$.

In order to get an upper and lower bound for $A_{2,0}^{u-d}$ and $B_{2,0}^{u-d}$ we randomly sample fit range choices (to estimate the systematical error). For each sample we fit the bootstrap ensemble (to estimate the statistical error). Then we generate the distributions of the fit parameters $\vec{\Theta}_{A}$ and $\vec{\Theta}_{B}$ which contain the combined error. In the last step we draw fit parameters from the distributions $\vec{\Theta}_{A}$ and $\vec{\Theta}_{B}$ and evaluate eq. (6.1) and eq. (6.2) with $t=0 \mathrm{GeV}$ and fixed pion mass $m_{\pi}$. This yields distributions for $A_{2,0}^{u-d}\left(t=0, m_{\pi}\right), B_{2,0}^{u-d}\left(t=0, m_{\pi}\right)$ and $J^{u-d}\left(m_{\pi}\right)=\left(A_{2,0}^{u-d}\left(t=0, m_{\pi}\right)+B_{2,0}^{u-d}\left(t=0, m_{\pi}\right)\right) / 2$.

We define the lower and upper error as 0.16 and 0.84 quantiles respectively. By repeating this process for many pion masses $m_{\pi}$ we are able to draw error bands as shown in the left panel of fig. 2 while the right panel shows the outcome for $m_{\pi}=m_{\pi}^{\text {phy }}$.

\section{Lattice results for the Axial-GFFs $\tilde{A}_{2,0}^{u-d}$ and $\tilde{B}_{2,0}^{u-d}$}

We carefully study fit range dependencies (induced by the combined fit to two- and threepoint functions). The error bars in fig. 3 show the (statistic and systematic) one-sigma error. Each single error bar is constructed from a histogram with sample-size $\mathrm{s}=$ (\# of fit range combinations) . (\#number of bootstrap ensembles). Here we have used $s=75 \cdot 300=22500$. A more detailed analysis will be published in the near future. 

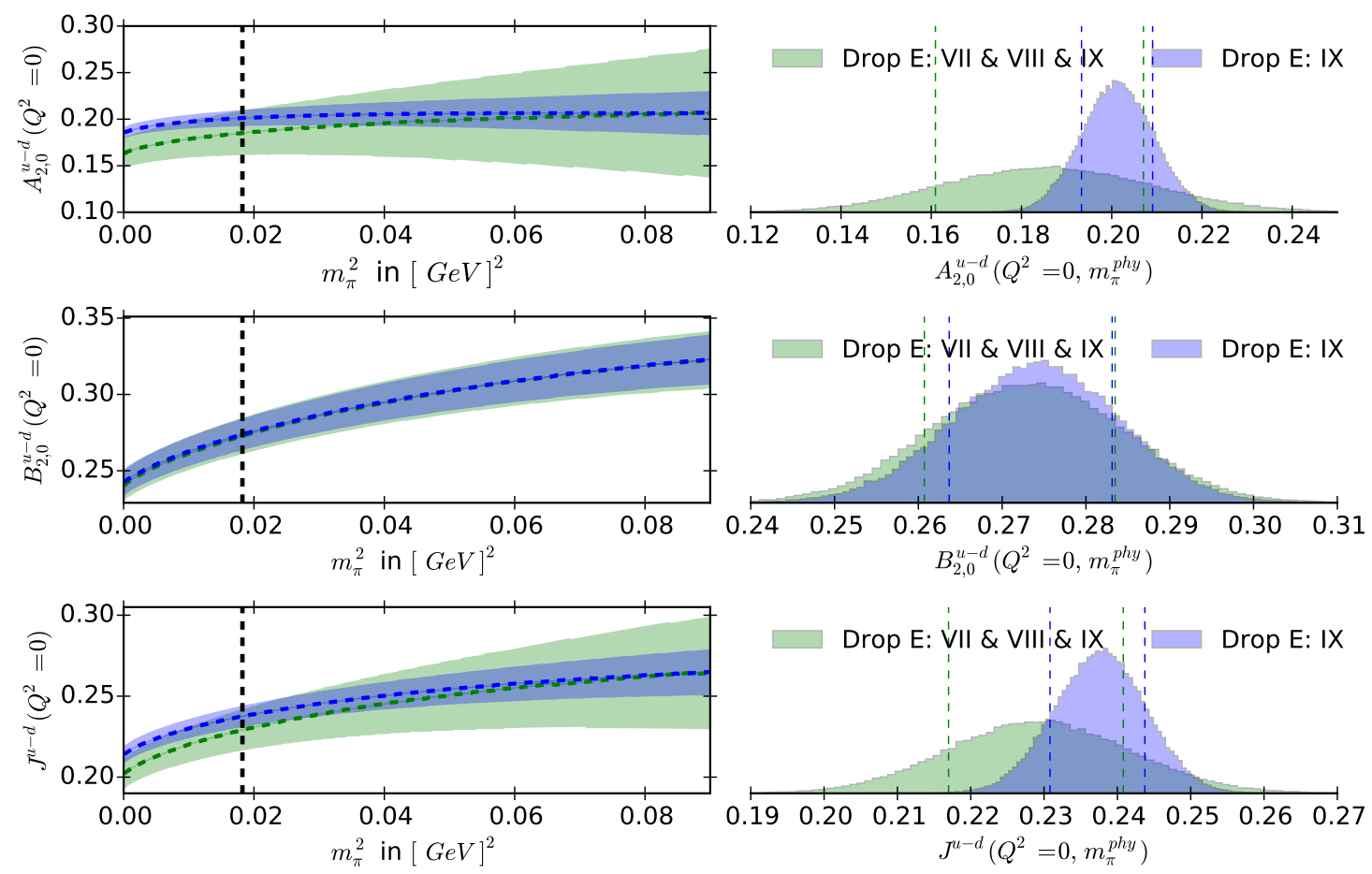

Figure 2: Results for $A_{2,0}^{u-d}$ and $B_{2,0}^{u-d}$ and $J^{u-d}$. The dashed black line indicates the physical pion mass. We always exclude the heaviest ensemble IX due to the applicability of BChPT (blue). To probe the stability of our Ansatz, we also show results where we additionally drop the two lightest ensembles VII \& VIII (green).

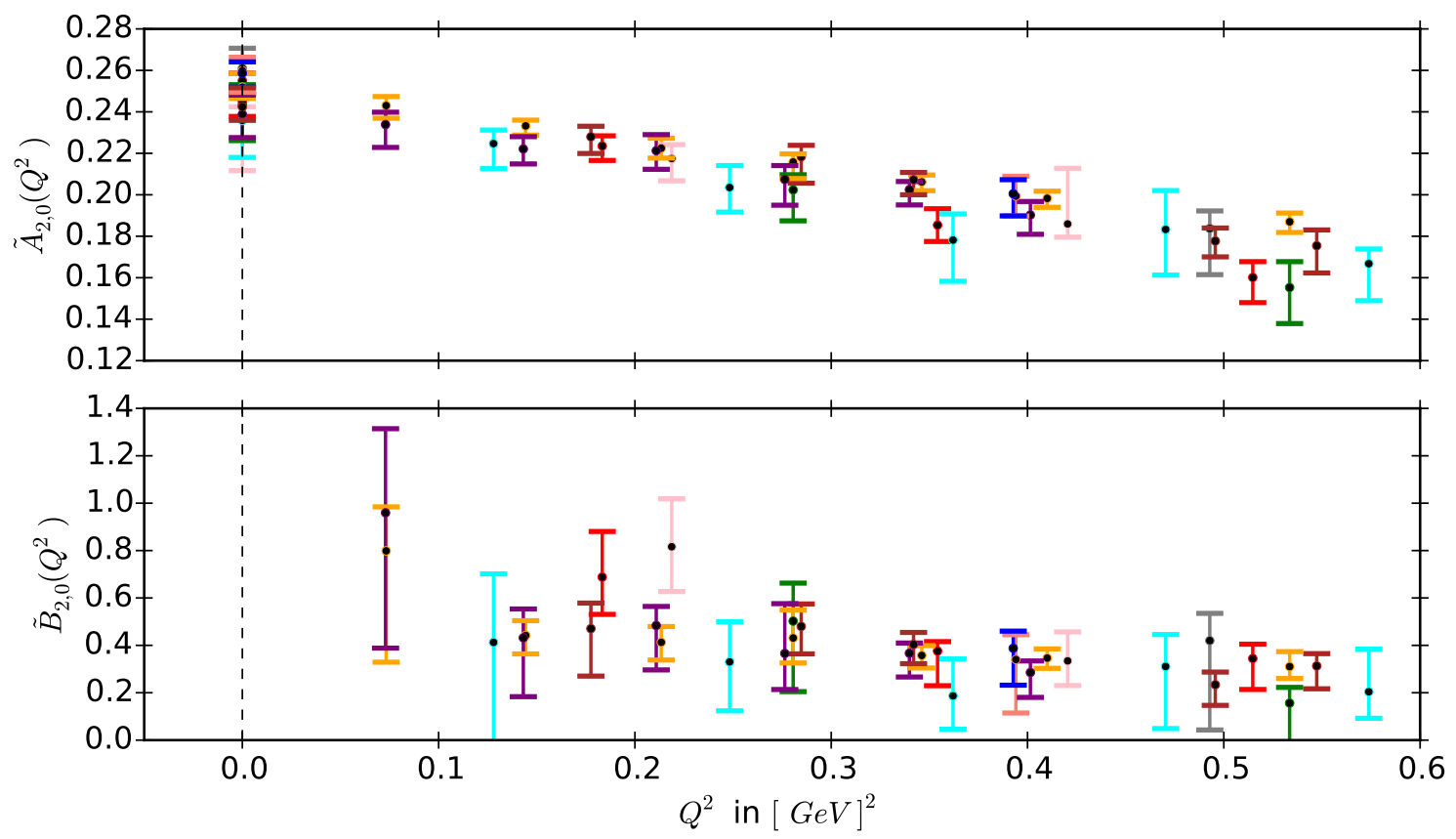

Figure 3: Lattice results for $\tilde{A}_{2,0}^{u-d}$ and $\tilde{B}_{2,0}^{u-d}$. For both GFFs we do not see a strong volume or pion mass dependence. Most notably are the small errors of ensemble VI with a $64^{3} \times 64$ volume and $L m_{\pi}=6.71$. 


\section{Conclusion}

We determine $A_{2,0}^{u-d}, B_{2,0}^{u-d}$ and $J^{u-d}$ by virtue of lattice QCD. Further, utilizing Baryon Chiral Perturbation Theory, we calculate the observables at the physical pion mass, where, we focused on the construction of reliable errors. This is achieved by the histogram method which propagates the statical error and the systematic error of the fit range choice. The final values are shown in table 2 .

\begin{tabular}{ll}
\hline Observable & $\overline{\mathrm{MS}} \mu=2 \mathrm{GeV}$ \\
\hline$A_{2,0}^{u-d}\left(Q^{2}=0, m_{\pi}^{p h y}\right)$ & $0.200(-7 /+9)$ \\
$B_{2,0}^{u-d}\left(Q^{2}=0, m_{\pi}^{p h y}\right)$ & $0.275(-11 /+8)$ \\
$J^{u-d}\left(m_{\pi}^{p h y}\right)$ & $0.238(-8 /+5)$ \\
\hline
\end{tabular}

Table 2: Table of results: The numbers correspond to the blue distributions shown in fig. 2. They have a systematic error from the application of Baryon Chiral Perturbation Theory to our lattice ensembles.

Further, we determine $\tilde{A}_{2,0}^{u-d}$ and $\tilde{B}_{2,0}^{u-d}$ which are the GFFs corresponding to the Axial-GPD, however, more work has to be done especially to extrapolate $\tilde{B}_{2,0}^{u-d}$ to $Q^{2}=0$.

Acknowledgements

This work is funded by Deutsche Forschungsgemeinschaft (DFG) within the transregional collaborative research centre 55 (SFB-TRR55). We acknowledge computer time on SuperMUC at the Leibniz Rechenzentrum in Garching.

\section{References}

[1] D. Müller, D. Robaschik, B. Geyer, F. M. Dittes, and J. Hořejši, "Wave functions, evolution equations and evolution kernels from light ray operators of QCD,” Fortsch. Phys., vol. 42, pp. 101-141, 1994.

[2] X.-D. Ji, "Gauge-Invariant Decomposition of Nucleon Spin,” Phys. Rev. Lett., vol. 78, pp. 610-613, 1997.

[3] X.-D. Ji, “Off forward parton distributions,” J. Phys., vol. G24, pp. 1181-1205, 1998.

[4] X.-D. Ji, “Deeply virtual Compton scattering,” Phys. Rev., vol. D55, pp. 7114-7125, 1997.

[5] LHPC, P. Hagler, J. W. Negele, D. B. Renner, W. Schroers, T. Lippert, and K. Schilling, “Transverse structure of nucleon parton distributions from lattice QCD,” Phys. Rev. Lett., vol. 93, p. 112001, 2004.

[6] M. Diehl, “Generalized parton distributions,” Phys. Rept., vol. 388, pp. 41-277, 2003.

[7] G. S. Bali et al., "Nucleon mass and sigma term from lattice QCD with two light fermion flavors," Nucl. Phys., vol. B866, pp. 1-25, 2013.

[8] G. S. Bali, S. Collins, B. Glässle, M. Göckeler, J. Najjar, R. H. Rödl, A. Schäfer, R. W. Schiel, W. Söldner, and A. Sternbeck, "Nucleon isovector couplings from $N_{f}=2$ lattice QCD," Phys. Rev., vol. D91, no. 5, p. 054501, 2015.

[9] P. Wein, P. C. Bruns, and A. Schäfer, "First moments of nucleon generalized parton distributions in chiral perturbation theory at full one-loop order," Phys. Rev., vol. D89, no. 11, p. 116002, 2014. 